\title{
A New Method to Calculate Water Film Stiffness and Damping for Water Lubricated Bearing with Multiple Axial Grooves
}

\author{
Guojun Ren*
}

\begin{abstract}
Water lubricated guide bearings for hydro turbines and pumps are conventionally designed with multiple axial grooves to provide effectively cooling and flushing away abrasives. Due to the variety of groove configuration in terms of number and size, a predication of their performance is difficult. This paper deals with an analytical procedure to investigate groove effect on load capacity, stiffness and damping for this type of bearing where it is considered as an assembly of many inclined slide bearings. The result can be applied to bearings made of hard materials combined with low bearing pressure.
\end{abstract}

Keywords: Water lubricated bearing, Groove effect, Stiffness and damping, Inclined slide bearing

\section{Introduction}

As we all known, water-lubricated guide bearings for hydro turbines and pumps are conventionally designed with multi-axial grooves. These grooves are provided for purpose of effectively cooling the bearing and flushing away abrasives. However, due to the variety of groove design in terms of its number and size, a prediction of bearing performance in terms of load capacity, stiffness and damping characteristics is very difficult. The author of this paper [1] introduced an analytical method to investigate groove effect on the Sommerfeld Number and coefficients of stiffness and damping based on inclined slide bearing solutions for bearings with rigid surface. However, the quality and accuracy of the solution depends on how close the geometry of the inclined slide bearing to represent the actual wedge shape at individual pads of the grooved bearing, especially the wedge shape of those pads which are loaded most. This paper examined three different geometric shapes of inclined slide bearings and provided a solution that would have satisfactory accuracy.

*Correspondence: garyr@thordonbearings.com

Thordon Bearings Inc., 3225, Burlington, ON L7M 1A6, Canada
A brief review of available literature is useful to understand the development of this method. For rigid surface plain bearings with no grooves, in terms of steady operation, besides the classic solution of long bearing theory by Sommerfeld [2] and short bearing theory by DuBois and Ocvirk [3], there are several excellent analytical solutions for finite length bearings. The finite length bearing theory by Childs et al. $[4,5]$ is one of the excellent solutions. Another good analytical solution is proposed by Capone et al. [6]. Numerical solutions with using finite difference and finite element methods are abundant. The evaluation of them is not the focus of this paper. For bearings designed with multi-axial grooves, Pai et al. [7] published a number of works on steady performance and dynamic stability of simple rotor. Ren [8] published a paper on calculation of water film thickness of water lubricated bearing with multi-axial grooves for steady state operation. On stiffness and damping coefficients of non-grooved plain bearings, classical short bearing solution is the most popular one. The solution by Childs et al. $[4,5]$ is strongly recommended for finite length bearings. It needs to mention that above works are related to rigid surface bearings. For deformable surface bearings, the effect of surface deformation is considered. Lahmar et al. [9] provides a procedure to simultaneously evaluate both 
static and dynamic performance with small perturbation method. Recent development was focused on CFD and FSI (fluid structure interaction) [10-17]. The effect of turbulence on stiffness and damping was investigated in Refs. $[18,19]$. In review on available information, methods to determine stiffness and damping are mainly relying on numerical simulation for bearings with multiple axial grooves. The objective of this paper was to provide a semi-analytical method to investigate the groove effect on load capacity, stiffness and damping based on infinite length and rigid surface which is approximately valid for bearings made of hard polymers under relatively low bearing pressure [10].

For the calculation results to be useful, certain conditions for bearings have to be applied. First of all, the ratio of bearing length to the width of bearing pad must be greater than 3.0 or higher. Secondly, the bearing pressure shall be relatively low so that the surface deformation effect doesn't overwhelmingly change the result. In spite of this paper doesn't include the effect of elastic deformation of bearing surface in terms of elastohydrodynamic lubrication, the results are valid approximately for polymer bearings with higher hardness and lower pressure that most pump and turbine guide bearings are the case. The result is not suitable to water lubricated bearings with rubber staves that needs special treatment either through experiment [20-29] or numerical analysis. Experimental study on bearings with multiple axial grooves demonstrates that a relatively rigid surface of bearing pad more easily forms hydrodynamic pressure than soft surface [22]. This has been demonstrated in the elastohydrodynamic study on sliding bearings [30]. A practice engineering application of stiffness and damping was shown in Ref. [31].

\section{Stiffness and Damping of Inclined Slide Bearings with Different Geometries}

The idea to evaluate the load capacity (Sommerfeld Number), the stiffness and damping coefficients of a circular bearing with multiple axial grooves is that the circular journal bearing can be considered as an assembly of many simple inclined slide bearings (Figure 1), so that the analytical results for an inclined slide bearing (Figure 2) can be used as building blocks to form a calculation method. Without loss of generality, Figure 1 demonstrates pressure created by bearing pad only. In reality, water pressure in grooves varies from negligible to significant. Since this procedure works with non-dimensional functions, the water pressure in grooves can be easily added back to dimensional pressure. The implementation of this idea starts from evaluating the dynamic characteristics of the sliding bearing shown in Figure 2. To avoid of confusion, an inclined slider is also called inclined slide bearing. A

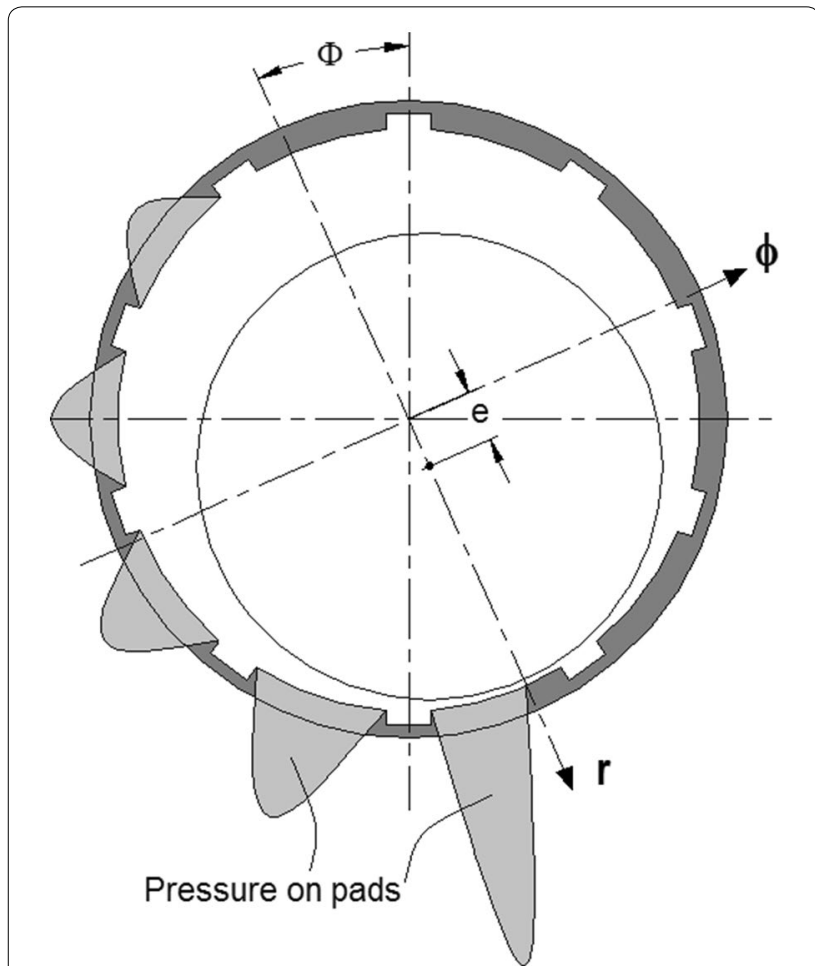

Figure 1 Grooved bearing as an assembly of sliding pads

sliding pad or bearing pads are always referring to the bearing surface between two neighboring grooves.

As indicated in the introduction, the load capacity of the entire bearing depends on the load capacity of the individual inclined sliding pads. The accuracy of the solution is a direct function of how the geometry of the inclined sliding bearing to represent the wedge shape of the individual pads of the circular bearing. In following sections, three useful geometries of inclined slide bearing are examined, the proximity to the wedge shape of the main bearing is compared.

\subsection{Linear Inclined Slide Bearing}

The linear inclined slider was used in previous work [1]. The water film is presented with a linear function as follows:

$$
h\left(x^{*}\right)=h_{T o} \cdot\left[1-x^{*} \cdot(\eta-1)\right] .
$$

The main functions of the solution are:

1. Load capacity (non-dimensional force)

$\Pi_{L}(\eta)=\frac{W_{o} \cdot h_{T 0}^{2}}{\mu \cdot V \cdot B^{2} \cdot L}=\frac{6 \cdot[(\eta+1) \cdot \ln \eta-2 \cdot(\eta-1)]}{(\eta-1)^{2} \cdot(\eta+1)}(2)$ 


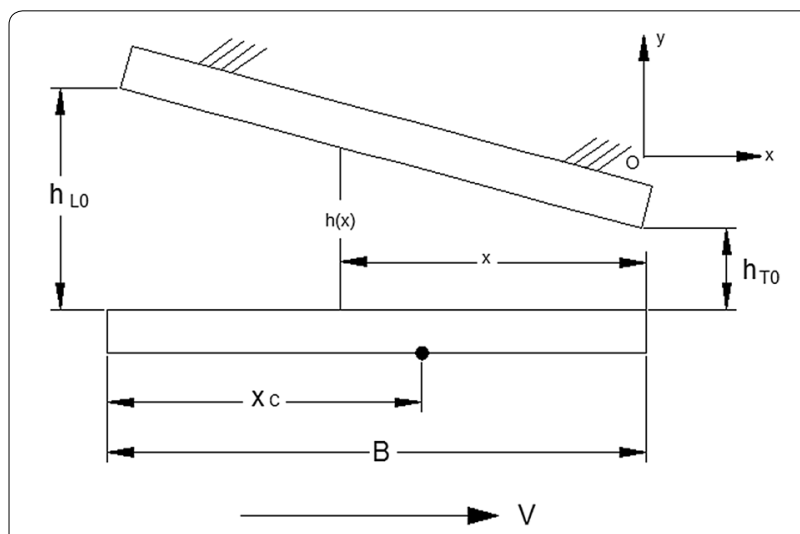

Figure 2 Infinite length inclined linear slide bearing

2. Location of static load center (non-dimensional distance)

$A_{L}(\eta)=\frac{x_{C}}{B}=\frac{\eta \cdot\left(\frac{\eta+2}{\eta-1}\right) \cdot \ln \eta-\frac{5}{2} \cdot(\eta-1)-3}{(\eta+1) \cdot \ln \eta-2(\eta-1)}$

3. Stiffness function (non-dimensional)

$K_{L}(\eta)=6 \cdot \frac{2 \cdot \eta \cdot \ln \eta-\eta+1}{\eta \cdot(\eta-1)^{2}}-\frac{6}{\eta \cdot(\eta+1)}+\frac{12}{1-\eta^{2}}$

4. Damping function (non-dimensional)

$C_{L}(\eta)=-6 \cdot \frac{\eta \cdot \ln \eta-\eta+1}{(\eta-1)^{3}}+6 \frac{\eta \cdot \ln \eta}{\left(\eta^{2}-1\right) \cdot(\eta-1)}$.

It is noticed that the right side of Eqs. (2)-(5) is a function of the ratio of film thickness at leading edge to the film thickness at trailing edge only. For the purpose of evaluating stiffness and damping, the film thickness at leading and trailing edges is considered as a function of time. This is shown in Figure 3.

The non-dimensional dynamic load is expressed by stiffness and damping function:

$$
\frac{W_{1} \cdot h_{T 0}^{2}}{\mu \cdot V \cdot B^{2} \cdot L}=-\left[K_{L}(\eta)+i \cdot C_{L}(\eta)\right] .
$$

According to Ref. [1], the final load on the linear inclined slide bearing is expressed with

$$
\begin{array}{r}
W=W_{0}-\frac{\mu \cdot V \cdot B^{2} \cdot L}{h_{T 0}^{3}} \cdot K_{L}(\eta) \cdot \Delta h_{T}(t) \\
-\frac{\mu \cdot B^{3} \cdot L}{h_{T 0}^{3}} \cdot C_{L}(\eta) \cdot \Delta \dot{h}_{T}(t) .
\end{array}
$$

Eq. (7) is the force of the individual slider under dynamic motion. It is the fundamental relationship between the slider force and slider displacement and squeeze velocity. As long as the four functions expressed from Eqs. (2)-(5) are known, the load capacity of the inclined slide bearing is fully defined. Therefore, the whole subject turns to finding a set of functions expressed in Eqs. (2)-(5) for different geometric shape of the slider.

\subsection{Exponential Gap Slide Bearing}

The shape of exponential gap slide bearing is expressed with following equation:

$$
h\left(x^{*}\right)=h_{T o} \cdot \exp \left(-x^{*} \cdot \ln \eta\right) .
$$

The four functions of result are as follows:

1. Non-dimensional load capacity

$$
\Pi_{E}(\eta)=\frac{W_{o} \cdot h_{T 0}^{2}}{\mu \cdot V \cdot B^{2} \cdot L}=\frac{\eta^{2}-1}{2 \eta^{2} \cdot(\ln \eta)^{2}}-\frac{3}{\left(\eta^{2}+\eta+1\right) \cdot \ln \eta},
$$

2. Location of static load center (non-dimensional distance)

$$
A_{E}(\eta)=\frac{x_{C}}{B}=\frac{\left(\eta^{2}+\eta+3\right) \cdot \eta^{2}-\frac{5(\eta+1)\left(\eta^{3}-1\right)}{6 \ln \eta}-3 \eta^{2} \ln \eta}{(\eta+1)\left(\eta^{3}-1\right)-6 \eta^{2} \cdot \ln \eta},
$$

3. Stiffness function (non-dimensional)

$$
K_{E}(\eta)=\frac{\eta^{2}-1}{\eta^{2} \cdot(\ln \eta)^{2}}-\frac{6}{\left(\eta^{2}+\eta+1\right) \cdot \ln \eta},
$$

4. Damping function (non-dimensional)

$$
C_{E}(\eta)=\frac{\eta^{2}-1}{\eta^{2}(\ln \eta)^{3}}-\frac{6}{\left(\eta^{2}+\eta+1\right) \cdot(\ln \eta)^{2}} .
$$

\subsection{Parabolic Gap Slide Bearing}

The author of this paper derived the four functions for a parabolic gap slider (see Appendix). The film thickness expression is presented by:

$$
h\left(x^{*}\right)=h_{T o} \cdot\left[1+x^{* 2} \cdot(\eta-1)\right] .
$$

The resulting functions are as follows.

1. Non-dimensional load capacity 


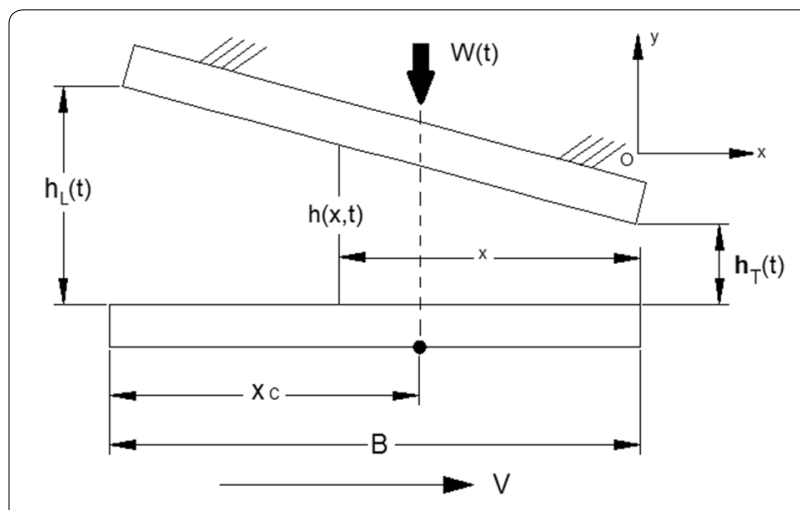

Figure 3 Inclined linear slide bearing in dynamic motion
It is to notice that the stiffness function of the parabolic gap and exponential gap is higher than the linear gap for all leading to trailing edge film ratios. This implied if the parabolic function is in good agreement with the actual bearing clearance (pads), a circular bearing simulated with the parabolic function will have a higher load capacity. The dynamic load center is slightly different from static load center Figure 4(d). Appendix provides the definition of load center ratio $R(\eta)$. This paper used static load center for Sommerfeld number evaluation and dynamic load center for stiffness and damping evaluation for all three types of sliding bearings.

\section{Assembly Procedure}

To evaluate which type of slider geometry best suitable

$$
\Pi_{P}(\eta)=\frac{W_{o} \cdot h_{T 0}^{2}}{\mu \cdot V \cdot B^{2} \cdot L}=\frac{\sqrt{\eta-1}+(\eta-2) \cdot \tan ^{-1} \sqrt{\eta-1}}{\eta^{2} \cdot \tan ^{-1} \sqrt{\eta-1}+\left(\eta+\frac{2}{3}\right) \cdot \sqrt{\eta-1}} .
$$

2. Location of static load center (non-dimensional distance)

$$
\begin{gathered}
A_{P}(\eta)=\frac{x_{C}}{B}=1+\frac{\int_{-1}^{0} x \cdot p_{o}^{*}(x, \eta) \mathrm{d} x}{\Pi_{p}(\eta)}, \\
p_{o}^{*}\left(x^{*}, \eta\right)=\frac{2}{\eta^{2} \tan ^{-1} \sqrt{\eta-1}+\left(\eta+\frac{2}{3}\right) \sqrt{\eta-1}} \times\left[\operatorname { t a n } ^ { - 1 } \left(x^{*} .\right.\right. \\
\text { 3. Stiffness function (non-dimensional) } \\
K_{P}(\eta)=2 \frac{\sqrt{\eta-1}+(\eta-2) \cdot \tan ^{-1} \sqrt{\eta-1}}{\eta^{2} \cdot \tan ^{-1} \sqrt{\eta-1}+\left(\eta+\frac{2}{3}\right) \cdot \sqrt{\eta-1}} .
\end{gathered}
$$

for building the circular bearing, the assembly procedure must be presented first. The first step of the assembly procedure is to define the location angles of each pad relative to a rotating co-ordinate frame $r-\phi$ [8]. Figure 5 shows a circular journal bearing with multiple axial grooves under a steady operational condition. By given load and shaft speed, the shaft center is offset from bear-

$$
p_{o}^{*}\left(x^{*}, \eta\right)=\frac{2}{\eta^{2} \tan ^{-1} \sqrt{\eta-1}+\left(\eta+\frac{2}{3}\right) \sqrt{\eta-1}} \times\left[\tan ^{-1}\left(x^{*} \cdot \sqrt{\eta-1}\right)+\frac{x^{*}\left(x^{*^{2}}-1\right)(\eta-1)^{\frac{3}{2}}-\eta^{2} \cdot x^{*} \cdot \tan ^{-1} \sqrt{\eta-1}}{\left(1+(\eta-1) \cdot x^{* 2}\right)^{2}}\right] .
$$

4. Damping function (non-dimensional)

$$
\begin{aligned}
C_{P}(\eta)= & \frac{2 \cdot(2 \eta+1)}{3 \eta+2+\frac{3 \eta^{2}}{\sqrt{\eta-1}} \cdot \tan ^{-1} \sqrt{\eta-1}} \\
& \times\left[\frac{1}{\eta}+\frac{3 \cdot \tan ^{-1} \sqrt{\eta-1}}{\sqrt{\eta-1}}\right]-\frac{4 \eta-1}{\eta \cdot(\eta-1)}+\frac{3 \cdot \tan ^{-1} \sqrt{\eta-1}}{(\eta-1)^{\frac{3}{2}}} .
\end{aligned}
$$

ing center with an eccentricity of " $e$ ". The connecting line between bearing center and shaft center is in-line with $r$-axis of the rotating coordinate system $r-\phi$. Assuming the bearing is fixed in position and the load is vertical as shown on the figure, the $r-\phi$ coordinate system has an attitude angle " $\Phi$ " with respect to the loading direction, namely, the $y$-axis. The attitude angle changes depending
Figure 4 shows the basic functions for these three inclined slide bearings. Since the non-dimensional load capacity function is exactly the half of the stiffness function for all type of geometries, it was not repeated in Figure 4. on load, shaft speed and groove numbers.

A second co-ordinate frame $x-y$ is defined in line with load direction. In this system, $y$-axis is in load direction and $x$-axis is perpendicular to the load direction.

In Figure 5, the $r$-axis divides the entire bearing into two equal halves. All pads underneath $r$-axis (in the sense 
of Figure 5) have convergent angles in shaft rotating direction and therefore are able to create hydrodynamic lifting forces. All pads above $r$-axis have divergent angles in shaft rotating direction and are therefore not able to create hydrodynamic lifting forces. In theory, the divergent bearing half could create a vacuum and therefore a negative pressure. However, since in practice, almost in all the cases, outside source of lubricant will be supplied to the bearing grooves, the divergent half will not create a negative pressure, but keep the same level of pressure as supplied lubricant as in grooves. It is therefore acceptable to assume the pressure on that half of bearing as zero. This assumption is corresponding to half Sommerfeld or Gumbel boundary condition.

Since the bearing is assumed to be fixed, all angles $\left(\alpha_{L i}\right.$, $\left.\alpha_{T i}=1,2,3, \ldots, N / 2\right)$ defining the positions of grooves will change with attitude angle which is an unknown parameter. One set of groove location angles only defines a particular equilibrium of steady operation. For calculation purpose, a set of "floating numbers" are assigned to the pads underneath the $r$-axis. As a rule, no matter how the attitude angle to change, it is always the first convergent pad underneath $r$-axis at the minimum water film location is assigned number " 1 ". Other pads are enumerated clockwise with number $2,3,4, \ldots$ in sequence.

After having defined the pad location angles, the film thickness ratio of leading to trailing edge under steady operation condition is

$$
\eta_{i}=\frac{1+\varepsilon \cdot \cos \alpha_{L i}}{1+\varepsilon \cdot \cos \alpha_{T i}}, \quad i=1,2, \ldots, N / 2 .
$$

The film thickness at any leading and trailing edge is

$$
\begin{array}{ll}
h_{L 0, i}=c \cdot\left(1+\varepsilon \cdot \cos \alpha_{L i}\right), & i=1,2, \ldots, N / 2, \\
h_{T 0, i}=c \cdot\left(1+\varepsilon \cdot \cos \alpha_{T i}\right) & i=1,2, \ldots, N / 2 .
\end{array}
$$

The second step of the assembly procedure is to calculate the force contribution of each pad to support the entire bearing load. Figure 6 illustrates the supporting force from one pad with location angles $\alpha_{L i}$ and $\alpha_{T i}$. Considering Eqs. (19) and (20), the force by each individual pad can be calculated with using Eq. (7) obtained from previous section. All terms in Eq. (21) are referred to pad number " $i$ ":

$$
W_{i}=W_{0 i}-\frac{c_{1} \cdot K\left(\eta_{i}\right)}{\left(1+\varepsilon \cdot \cos \alpha_{T i}\right)^{3}} \cdot \Delta h_{T i}(t)-\frac{c_{2} \cdot C\left(\eta_{i}\right)}{\left(1+\varepsilon \cdot \cos \alpha_{T i}\right)^{3}} \cdot \Delta \dot{h}_{T i}(t),
$$

with $c_{1}=\frac{\mu \cdot V \cdot B^{2} \cdot L}{c^{3}} ; \quad c_{2}=\frac{\mu \cdot B^{3} \cdot L}{c^{3}}$.

In Figure 6, the pad load is considered to be in direction pointing to bearing center. The projection of bearing load to $r-\phi$ co-ordinate system is then

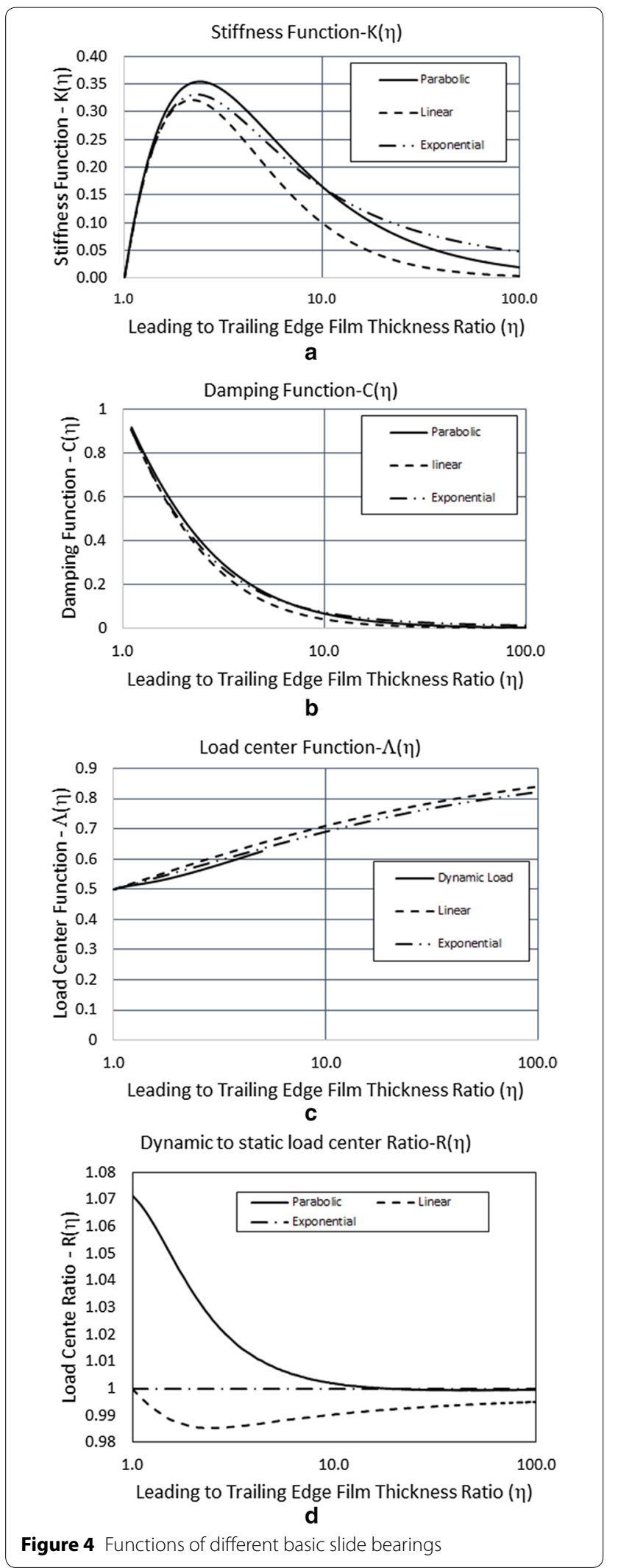




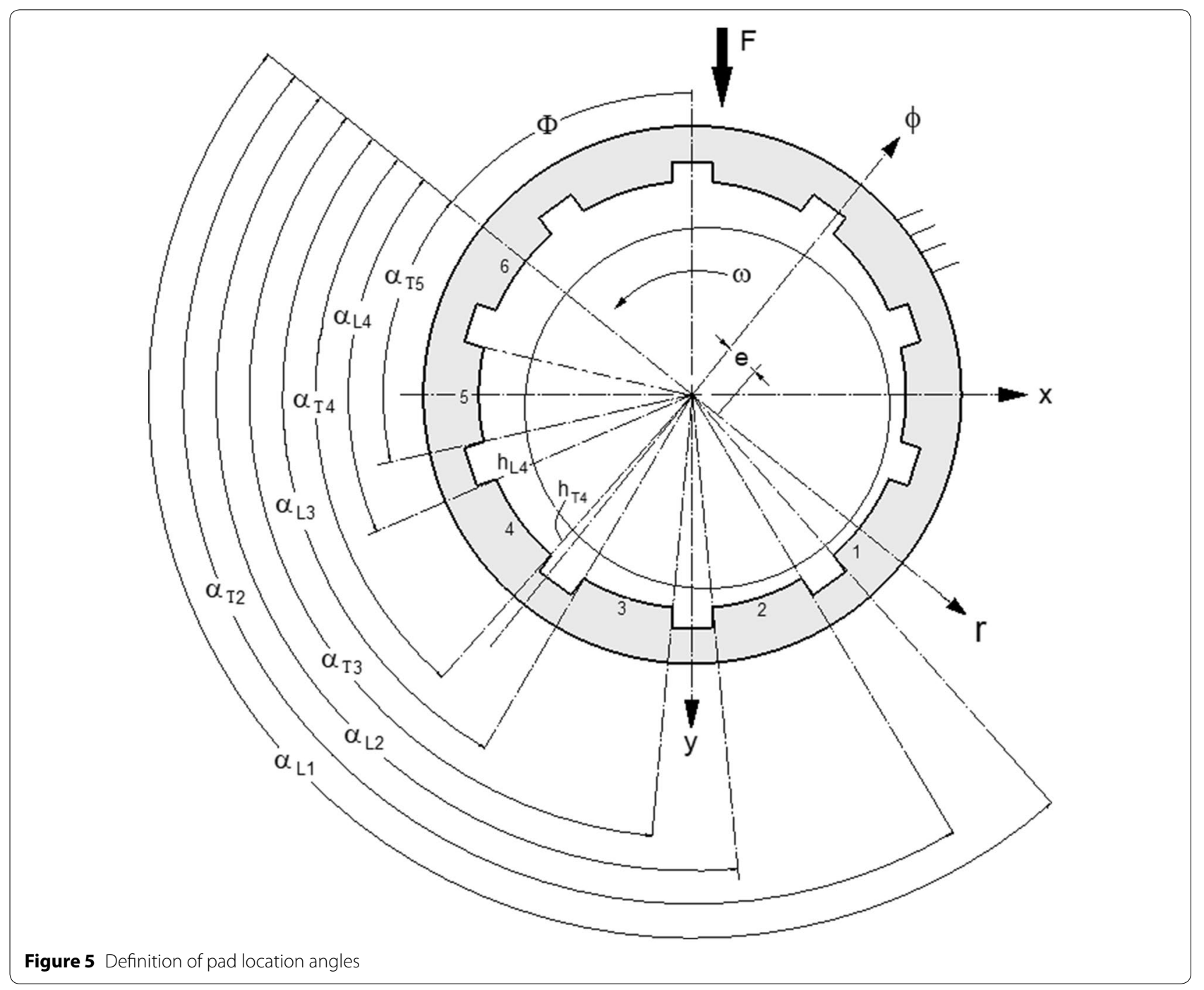

$$
\begin{aligned}
& -W_{i, r}=W_{0 i} \cos \left(\pi-\Theta_{i}\right) \\
& -\left(\frac{c_{1} \cdot K\left(\eta_{i}\right)}{\left(1+\varepsilon \cdot \cos \alpha_{T i}\right)^{3}} \cdot \Delta h_{T i}(t)+\frac{c_{2} \cdot C\left(\eta_{i}\right)}{\left(1+\varepsilon \cdot \cos \alpha_{T i}\right)^{3}} \cdot \Delta \dot{h}_{T i}(t)\right) \cdot \cos \left(\pi-\vartheta_{i}\right), \\
& W_{i, \varphi}=W_{0 i} \sin \left(\pi-\Theta_{i}\right) \\
& -\left(\frac{c_{1} \cdot K\left(\eta_{i}\right)}{\left(1+\varepsilon \cdot \cos \alpha_{T i}\right)^{3}} \cdot \Delta h_{T i}(t)+\frac{c_{2} \cdot C\left(\eta_{i}\right)}{\left(1+\varepsilon \cdot \cos \alpha_{T i}\right)^{3}} \cdot \Delta \dot{h}_{T i}(t)\right) \cdot \sin \left(\pi-\vartheta_{i}\right),
\end{aligned}
$$

In Eqs. (22), (23), the function $K(\eta)$ is one of the function $K_{L}(\eta), K_{E}(\eta)$ or $K_{P}(\eta)$ depending on which one is chosen. The same applied to function $C(\eta)$. For the entire circular bearing, the dynamic part of pad force is only caused by a very small change of bearing eccentricity $\Delta e$ and attitude angle $\Delta \Phi$, the film thickness at trailing edge can be correlated to these small eccentricity and attitude angle change as follows

$$
\Delta h_{T i}=\cos \alpha_{T i} \cdot \Delta e+\sin \alpha_{T i} \cdot e \cdot \Delta \Phi .
$$

The same can be applied to the pad velocity. However, the velocity must refer to the entire pad, not just the 


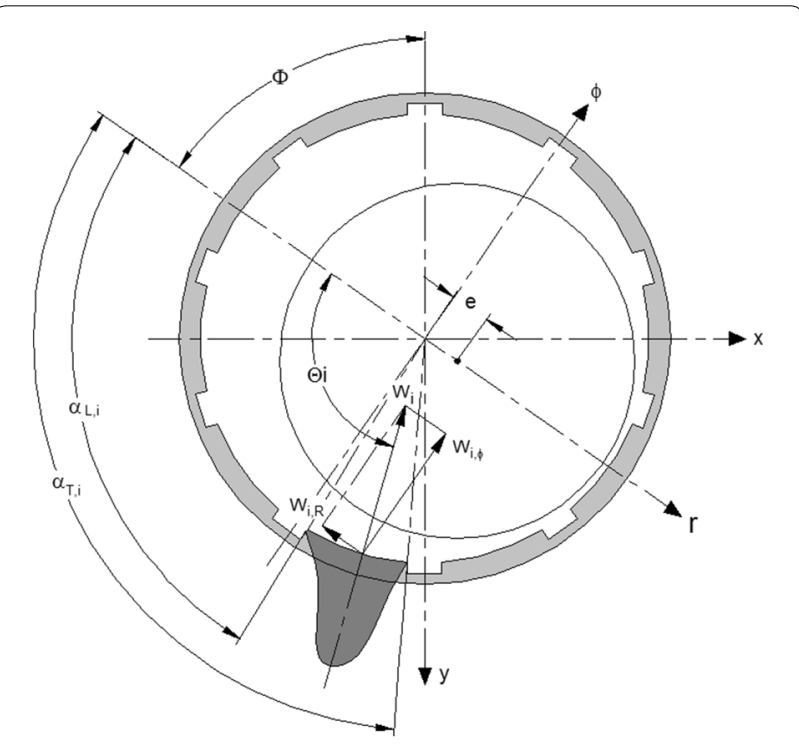

Figure 6 Contribution of pad load to bearing

trailing edge. This implies that the pad has no rotation about its load center at any instant of dynamic motion. Therefore the velocity becomes:

$$
\Delta \dot{h}_{T i}=\cos \left(\pi-\Theta_{i}\right) \cdot \Delta \dot{e}+\sin \left(\pi-\vartheta_{i}\right) \cdot e \cdot \Delta \dot{\Phi}
$$

$$
\begin{aligned}
& K_{\phi r, i}=\frac{\cos \alpha_{T i}}{\left(1+\varepsilon \cdot \cos \alpha_{T i}\right)^{3}} \cdot K\left(\eta_{i}\right) \cdot \sin \left(\pi-\vartheta_{i}\right), \\
& K_{\phi \phi, i}=\frac{\sin \alpha_{T i}}{\left(1+\varepsilon \cdot \cos \alpha_{T i}\right)^{3}} \cdot K\left(\eta_{i}\right) \cdot \sin \left(\pi-\vartheta_{i}\right), \\
& C_{r r, i}=\frac{C\left(\eta_{i}\right)}{\left(1+\varepsilon \cdot \cos \alpha_{T i}\right)^{3}} \cdot \cos ^{2}\left(\pi-\vartheta_{i}\right), \\
& C_{r \phi, i}=C_{\phi r, i}=\frac{C\left(\eta_{i}\right)}{\left(1+\varepsilon \cdot \cos \alpha_{T i}\right)^{3}} \cdot \cos \left(\pi-\vartheta_{i}\right) \cdot \sin \left(\pi-\vartheta_{i}\right), \\
& C_{\phi \phi, i}=\frac{C\left(\eta_{i}\right)}{\left(1+\varepsilon \cdot \cos \alpha_{T i}\right)^{3}} \cdot \sin ^{2}\left(\pi-\vartheta_{i}\right) .
\end{aligned}
$$

\section{Quality Comparison of Slide Bearing Geometry}

Above section proposed an idea of using an array of inclined slide bearings to build a circular journal bearing with multiple axial grooves. However, the quality of this approach depends on how closely the individual slider represents the shape of individual pad at any given location and eccentricity ratio.

In true sense of grooved bearing, the non-dimensional form of water film thickness at any given location angle " $\alpha$ " (Figure 7) is

$$
\theta_{i}=A_{d}\left(\eta_{i}\right) \cdot \alpha_{T i}+\left[1-A_{d}\left(\eta_{i}\right)\right] \cdot \alpha_{L i}, \quad \Theta_{i}=A\left(\eta_{i}\right) \cdot \alpha_{T i}+\left[1-A\left(\eta_{i}\right)\right] \cdot \alpha_{L i}, \quad i=1,2,3, \ldots, N / 2 .
$$

The function $A(\eta)$ is one of the $A_{L}(\eta), A_{E}(\eta)$ or $A_{P}(\eta)$ depending on which type of sliding bearing chosen and $A_{d}(\eta)$ is one of the $A_{L d}(\eta), A_{E d}(\eta)$ or $A_{P d}(\eta)$ depending on which type of sliding bearing chosen. Inserting Eqs. (24) and (25) into Eqs. (22) and (23), their matrix form can be expressed as

$$
\bar{h}=\frac{h}{c}=(1+\varepsilon \cdot \cos \alpha)
$$

When evaluating the individual inclined slide bearing, the non-dimensional water film thickness is expressed as

$$
\begin{aligned}
\left(\begin{array}{c}
-W_{i, r} \\
W_{i, \phi}
\end{array}\right)= & \left(\begin{array}{c}
W_{o i} \cdot \cos \left(\pi-\Theta_{i}\right) \\
W_{0 i} \cdot \sin \left(\pi-\Theta_{i}\right)
\end{array}\right)-c_{1}\left[\begin{array}{cc}
K_{r r, i} & K_{r \phi, i} \\
K_{\phi r, i} & K_{\phi \phi, i}
\end{array}\right]\left(\begin{array}{c}
\Delta e \\
e \cdot \Delta \phi
\end{array}\right) \\
& -c_{2}\left[\begin{array}{cc}
C_{r r, i} & C_{r \phi, i} \\
C_{\phi r, i} & C_{\phi \phi, i}
\end{array}\right]\left(\begin{array}{c}
\Delta \dot{e} \\
e \Delta \dot{\phi}
\end{array}\right) .
\end{aligned}
$$

The coefficients of stiffness and damping for the pad with index " $i$ " are:

$$
\begin{aligned}
K_{r r, i} & =\frac{\cos \alpha_{T i}}{\left(1+\varepsilon \cdot \cos \alpha_{T i}\right)^{3}} \cdot K\left(\eta_{i}\right) \cdot \cos \left(\pi-\vartheta_{i}\right), \\
K_{r \phi, i} & =\frac{\sin \alpha_{T i}}{\left(1+\varepsilon \cdot \cos \alpha_{T i}\right)^{3}} \cdot K\left(\eta_{i}\right) \cdot \cos \left(\pi-\vartheta_{i}\right),
\end{aligned}
$$

a function of the film thickness ratio of leading edge to trailing edge as well as the local coordinate " $s$ " (Figure 7). Therefore, the non-dimensional water film thickness for the pad number " $i$ " in terms of above mentioned water film thickness ratio and local coordinate can be presented in following form: 


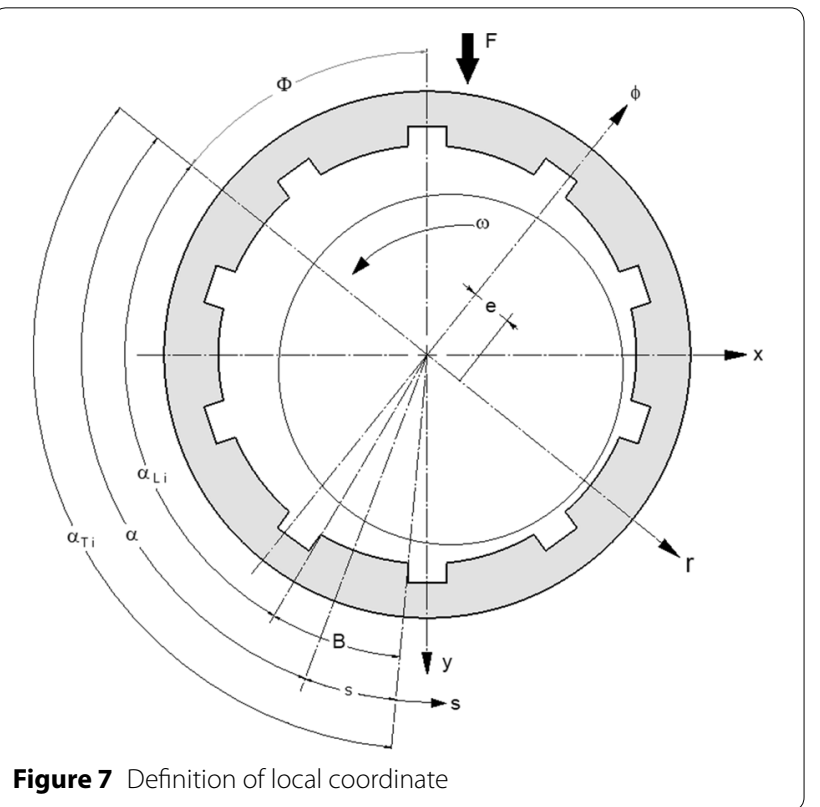

is $\left(1+\varepsilon \cdot \cos \alpha_{T i}\right) \cdot \eta_{i}$ when $\bar{s}=-1$. To evaluate which function from Eqs. (29)-(31) is the best approach to Eq. (28), one set of square root errors was defined. These are:

$$
S_{L}(i, \varepsilon)=\frac{\sqrt{\int_{-1}^{0}\left[\bar{h}(s, i)-\bar{h}_{L}(s, i)\right]^{2} \mathrm{~d} s}}{\int_{-1}^{0} \bar{h}(s, i) \cdot \mathrm{d} s},
$$

$$
S_{P}(i, \varepsilon)=\frac{\sqrt{\int_{-1}^{0}\left[\bar{h}(s, i)-\bar{h}_{P}(s, i)\right]^{2} \mathrm{~d} s}}{\int_{-1}^{0} \bar{h}(s, i) \cdot \mathrm{d} s} .
$$

$$
\bar{h}(\bar{s}, i)=\left\{1+\varepsilon \cdot \cos \left[\bar{s} \cdot\left(\alpha_{T i}-\cos ^{-1} \frac{\left(1+\varepsilon \cdot \cos \alpha_{T i}\right) \cdot \eta_{i}-1}{\varepsilon}\right)+\alpha_{T i}\right]\right\},
$$

Where $\bar{s}=s / B$ is non-dimensional local coordinate. The true bearing may have grooves with chamfer or round fillet. Since any chamfer and fillet will be too large for water film formation. Therefore, chamfer and fillet must be considered as part of grooves, not surface of pads. All other expression of water film thickness for bearing pad " $i$ " can also expressed with local coordinate and the water film thickness ratio at leading and trailing edge.

For linear inclined slide bearing

$$
\bar{h}_{L}(\bar{s}, i)=\left(1+\varepsilon \cdot \cos \alpha_{T i}\right) \cdot\left[1-\bar{s} \cdot\left(\eta_{i}-1\right)\right] .
$$

For exponential inclined slide bearing

$$
\bar{h}_{E}(\bar{s}, i)=\left(1+\varepsilon \cdot \cos \alpha_{T i}\right) \cdot \exp \left[-\bar{s} \cdot \ln \eta_{i}\right] .
$$

For parabolic inclined slide bearing

$$
\bar{h}_{P}(\bar{s}, i)=\left(1+\varepsilon \cdot \cos \alpha_{T i}\right) \cdot\left[1+\bar{s}^{2} \cdot\left(\eta_{i}-1\right)\right] .
$$

Eqs. (29)-(31) presents the water film thickness that are intended to be used to replace Eq. (28). The purpose of doing so is to simplify the problem by solving Reynold's Equation at the inclined slide bearing level rather than at the full bearing level. It is to notice that all these equations return the film thickness at trailing edge of pad " $i$ " which is $\left(1+\varepsilon \cdot \cos \alpha_{T i}\right)$ when $\bar{s}=0$. By the same token, they return the film thickness at leading edge which
Eqs. (32)-(34) were derived from non-dimensional water film thickness and are function of eccentricity ratio, number and distribution of grooves. They are valid for any size bearings with any number of grooves. In following, a 12-groove bearing is examined for the square root errors. This bearing will have six bearing pads taking load. Assuming the location of the minimum film thickness falls into the very center of a groove, so that the first pad named with one will have the entire pad being loaded.

Figure 8 shows the result for the bearing with 12 grooves. It showed that the parabolic inclined slider has the least error for the first pad for eccentricity ratio from 0.9 to 0.999 which is the range of most interest for water lubricated guide bearings. The linear slider seems to be best suitable for second pad at high eccentricity ratio and rest of other pads. The exponential slider seems to be suitable for pads except for first one at lower eccentricity ratio. However, this is only observations from a bearing with 12 grooves. Investigation on different number of grooves showed that the error for exponential slider changes rapidly with increasing eccentricity ratio, meaning that for small eccentricity ratio, errors are small, for large eccentricity ratio, errors are big. This is especially true for first and second pad. The linear slide bearing is insensitive to eccentricity ratio. Therefore, in following evaluation, a scheme that parabolic slider to first pad and 
linear slider to rest of pads is applied. This paper investigated only three types of sliding bearings. It is certainly there must be other types of sliding bearings that would fit for the purpose. As demonstrated in Ref. [8], the first pad takes the most load of entire bearing. Even though the parabolic slider is only able to simulate the first loaded pad, it is still a significant improvement of load capacity in comparison to the approach with all linear sliders.

\section{Steady Operation and Sommerfeld Number}

\subsection{Effect of Groove Number}

Ref. [8] provides a procedure to calculate the load capacity and water film under steady operational condition. The non-dimensional load capacity of bearing was determined as follows:

$$
\begin{aligned}
W_{0 r, i} & =\frac{\Pi\left(\eta_{i}\right)}{\left(1+\varepsilon \cdot \cos \alpha_{T i}\right)^{2}} \cdot \cos \left\{\pi-\Theta_{i}\right\}, \\
W_{0 \varphi, i} & =\frac{\Pi\left(\eta_{i}\right)}{\left(1+\varepsilon \cdot \cos \alpha_{T i}\right)^{2}} \cdot \sin \left\{\pi-\Theta_{i}\right\},
\end{aligned}
$$

where

$$
\Pi\left(\eta_{i}\right)= \begin{cases}\Pi_{L}\left(\eta_{i}\right), & \text { for linear slider, } \\ \Pi_{P}\left(\eta_{i}\right), & \text { for parabolic slider }\end{cases}
$$

The total non-dimensional supporting force contributed by all pads underneath $r$-axis is then the sum of all components above:

$$
\begin{aligned}
& W_{0 \varphi}=\sum_{i=2}^{N / 2} W_{0 \varphi, i}+\lambda^{2} \cdot W_{0 \varphi, 1}, \\
& W_{0 r}=\sum_{i=2}^{N / 2} W_{0 r, i}+\lambda^{2} \cdot W_{0 r, 1},
\end{aligned}
$$

where $\lambda$ is a number less than 1.0. In Figure 5 , if the position of minimum film thickness is located within the pad number 1 , only a part of this pad will take load. The number " $\lambda$ " gives the percentage of the pad that takes load. Its value is unknown at beginning of a calculation. It depends on attitude angle " $\Phi$ ", width of bearing pads " $B$ ", width of grooves as well as the relationship between bearing loading direction and the pad position to which the load pointing to. For vertical bearings, such as hydro turbine guide bearing and vertical pump bearing, loading direction is undefined. In this case, practical calculation can be done by assuming $\lambda=0.5$ which presents a condition with least load capacity. For horizontal bearings, load direction can be easily defined. Parameter $\lambda$ can be determined by an iteration. At beginning, first to assume an initial value, for example 0.5 , then calculate the attitude angle according to Eq. (40) below and subsequently calculate the new $\lambda$-value. With new value run calculation again, get another attitude angle. Repeating the same procedure until a satisfactory result obtained. In evaluation on Figures 9, 10, 11 and 12, $\lambda=1.0$ was used. A full analysis of effect of $\lambda$-value on Sommerfeld Number is worth of a full separate paper to discuss.

The resultant force in dimensional form is then

$$
W_{0}=\frac{\mu \cdot V \cdot B^{2} \cdot L}{c^{2}} \sqrt{W_{0 \varphi}^{2}+W_{0 r}^{2}} .
$$

The attitude angle is:

$$
\Phi=-\tan ^{-1} \frac{W_{0 \phi}}{W_{0 r}} .
$$

According to conventional definition of Sommerfeld Number for circular bearings, it is

$$
S=\frac{\mu \cdot N_{s} \cdot d \cdot L}{W_{0}} \cdot\left(\frac{d}{2 c}\right)^{2} .
$$

The Sommerfeld Number for grooved bearings can be derived with using Eq. (39):

$$
S=\frac{d^{2}}{4 \cdot \pi \cdot B^{2} \cdot \sqrt{W_{0 \varphi}^{2}+W_{0 r}^{2}} .}
$$

The Sommerfeld Number, defined by Eq. (42) is a function of number of grooves and eccentricity ratio. Its reciprocal defines the load capacity of a bearing while the reciprocal of Eq. (41) is the actual load to the bearing. It is evident that for low eccentricity ratio (less than 0.9 ), there is no significant difference between the modeling with all linear sliders and the one with mixed sliders, namely, the first one using parabolic slider and rest of them linear sliders. However, for high eccentricity ratio $(\varepsilon>0.9)$, this reflects the case of high loading, the difference can be significant, Figure 9. The Sommerfeld Number simulated with all linear pads can be up to 5 times higher than the Sommerfeld Number with mixed pad geometry for the example investigated. By definition, higher Sommerfeld Number means lower load capacity. This is reflected in Figure 10.

The Sommerfeld Number ratio shown in Figure 10 is the Sommerfeld Number with all linear sliders divided by the Sommerfeld Number with mixed pads in which the first pad is parabolic and rest of them linear. Water lubricated guide bearings can be subject to eccentricity ratio as high as 0.999. In this case, an all linear modeling definitely under estimate the bearing loading capacity. Based on the quality comparison of sliding bearing geometries 


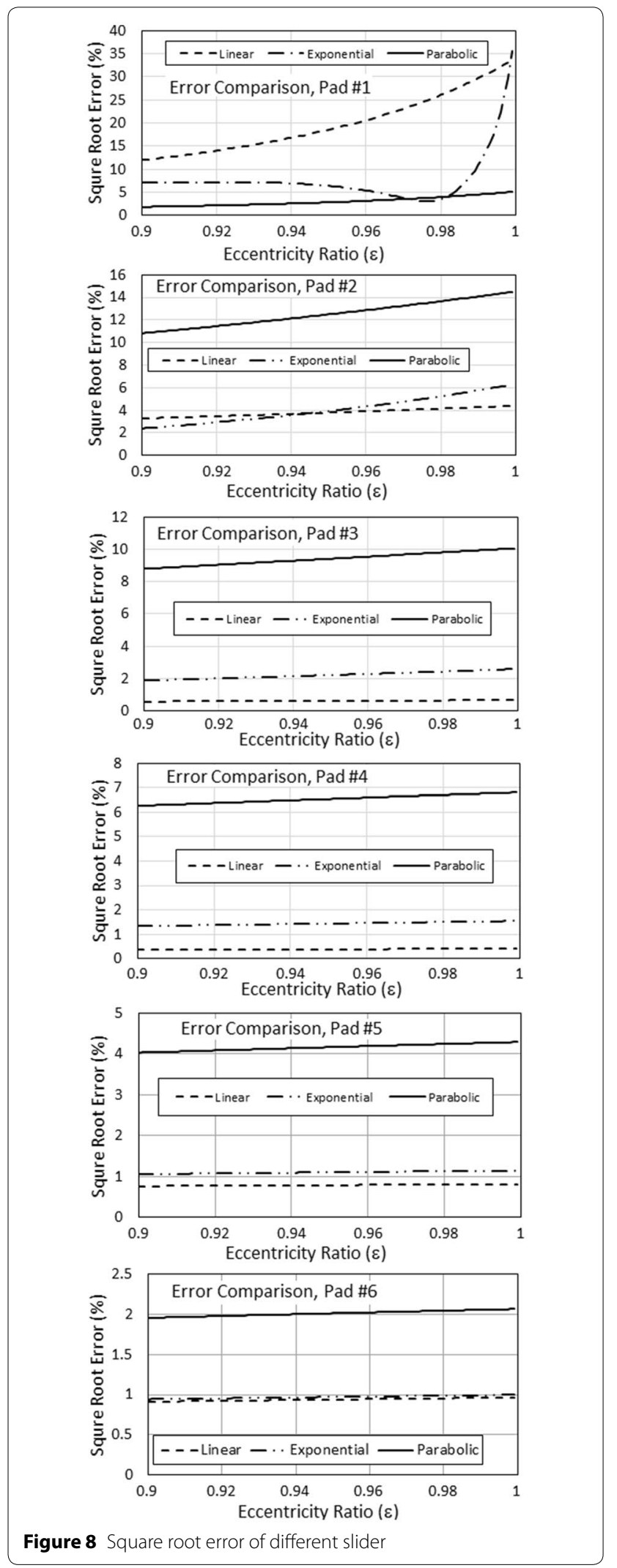

in previous section, the parabolic gap is more closed to true shape of bearing clearance of the first pad. Therefore, the mixed scheme must more closely present the true bearing performance. It is an improvement of all linear slider modeling, especially for large eccentricity ratio.

Ren et al. [1] in their previous paper quantitatively demonstrated that the load capacity of grooved bearing is lower than that of non-grooved bearings. The Sommerfeld Number of grooved bearing modeled with all linear pads was compared with the bench mark Sommerfeld Number, namely the formulation from Refs. $[4,5]$. A similar comparison is made here for the grooved bearing with mixed type of inclined sliders to the non-grooved bearings. The Sommerfeld Number of the solution by Childs is again used as bench mark for the comparison.

The massive curve on Figure 11 is the Sommerfeld Number of non-grooved bearing. Other curves are for grooved bearing with different number of grooves. Definitely, the grooves reduce the load capacity of a bearing. The more grooves, the bigger is the load capacity reduction.

The ratio of Sommerfeld Number for grooved to nongrooved bearing is shown in Figure 12. This provides a better visualization as how much the load capacity reduction can be expected. From Figure 12 is to see reducing the number of grooves is an effective way to increase load capacity of a grooved bearing.

\subsection{Effect of Groove Size}

From the defining equation of Sommerfeld Number of grooved bearing Eq. (42), it is a function of ratio $d / B$. For a fixed number of grooves, the size (width) of groove will take away a part of bearing surface which results in a narrower bearing pad. This increases the $d / B$ ratio. Therefore, for a real load capacity of a practice design, groove effect must be taken into consideration, especially grooves with round or fillet corners.

\section{Stiffness and Damping Coefficients}

Following the similar procedure in Refs. $[1,8]$, the nondimensional stiffness and damping coefficients for a circular bearing with multi-axial grooves were obtained by summarizing the coefficients of stiffness and damping over all supporting pads and are expressed as

$$
\begin{aligned}
K_{r r}= & \sum_{i=2}^{N / 2} \frac{\cos \alpha_{T i}}{\left(1+\varepsilon \cdot \cos \alpha_{T i}\right)^{3}} \cdot K_{L}\left(\eta_{i}\right) \cdot \cos \left(\pi-\vartheta_{i}\right) \\
& +\frac{\lambda^{2} \cdot \cos \alpha_{T 1}}{\left(1+\varepsilon \cdot \cos \alpha_{T 1}\right)^{3}} \cdot K_{P}\left(\eta_{1}\right) \cdot \cos \left(\pi-\vartheta_{1}\right),
\end{aligned}
$$




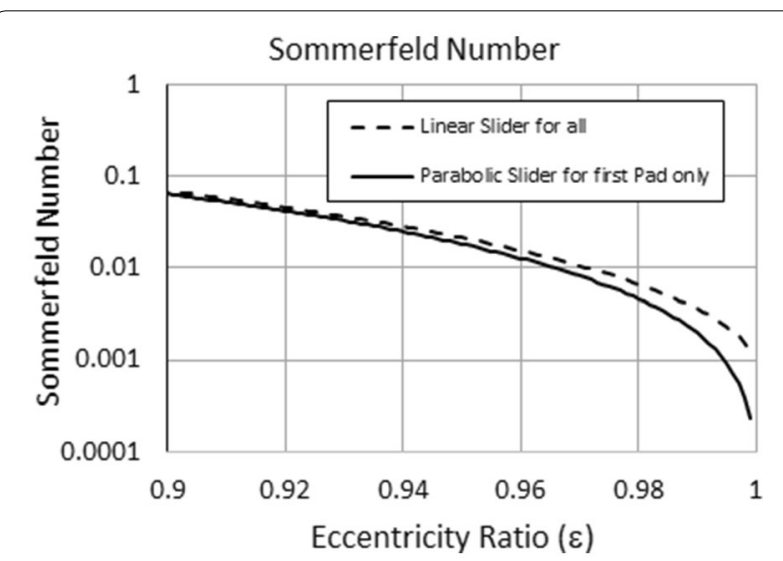

Figure 9 Sommerfeld Number comparison

$$
\begin{aligned}
K_{r \phi}= & \sum_{i=2}^{N / 2} \frac{\sin \alpha_{T i}}{\left(1+\varepsilon \cdot \cos \alpha_{T i}\right)^{3}} \cdot K_{L}\left(\eta_{i}\right) \cdot \cos \left(\pi-\vartheta_{i}\right) \\
& +\frac{\lambda^{2} \cdot \sin \alpha_{T 1}}{\left(1+\varepsilon \cdot \cos \alpha_{T 1}\right)^{3}} \cdot K_{P}\left(\eta_{1}\right) \cdot \cos \left(\pi-\vartheta_{1}\right), \\
K_{\phi r}= & \sum_{i=2}^{N / 2} \frac{\cos \alpha_{T i}}{\left(1+\varepsilon \cdot \cos \alpha_{T i}\right)^{3}} \cdot K_{L}\left(\eta_{i}\right) \cdot \sin \left(\pi-\vartheta_{i}\right) \\
& +\frac{\lambda^{2} \cdot \cos \alpha_{T 1}}{\left(1+\varepsilon \cdot \cos \alpha_{T 1}\right)^{3}} \cdot K_{P}\left(\eta_{1}\right) \cdot \sin \left(\pi-\vartheta_{1}\right),
\end{aligned}
$$

$$
\begin{aligned}
K_{\phi \phi}= & \sum_{i=2}^{N / 2} \frac{\sin \alpha_{T i}}{\left(1+\varepsilon \cdot \cos \alpha_{T i}\right)^{3}} \cdot K_{L}\left(\eta_{i}\right) \cdot \sin \left(\pi-\vartheta_{i}\right) \\
& +\frac{\lambda^{2} \cdot \sin \alpha_{T 1}}{\left(1+\varepsilon \cdot \cos \alpha_{T 1}\right)^{3}} \cdot K_{P}\left(\eta_{1}\right) \cdot \sin \left(\pi-\vartheta_{1}\right)
\end{aligned}
$$$$
C_{r r}=\sum_{i=2}^{N / 2} \frac{C_{L}\left(\eta_{i}\right)}{\left(1+\varepsilon \cdot \cos \alpha_{T i}\right)^{3}} \cdot \cos ^{2}\left(\pi-\vartheta_{i}\right)
$$$$
+\frac{\lambda^{3} \cdot C_{P}\left(\eta_{1}\right)}{\left(1+\varepsilon \cdot \cos \alpha_{T 1}\right)^{3}} \cdot \cos ^{2}\left(\pi-\vartheta_{1}\right) \text {, }
$$$$
C_{r \phi}=C_{\phi r}=\sum_{i=2}^{N / 2} \frac{C_{L}\left(\eta_{i}\right)}{\left(1+\varepsilon \cdot \cos \alpha_{T i}\right)^{3}} \cdot \cos \left(\pi-\vartheta_{i}\right) \cdot \sin \left(\pi-\vartheta_{i}\right)
$$$$
+\frac{\lambda^{3} \cdot C_{P}\left(\eta_{1}\right)}{\left(1+\varepsilon \cdot \cos \alpha_{T 1}\right)^{3}} \cdot \cos \left(\pi-\vartheta_{1}\right) \cdot \sin \left(\pi-\vartheta_{1}\right),
$$$$
C_{\phi \phi}=\sum_{i=2}^{N / 2} \frac{C_{L}\left(\eta_{i}\right)}{\left(1+\varepsilon \cdot \cos \alpha_{T i}\right)^{3}} \cdot \sin ^{2}\left(\pi-\vartheta_{i}\right)
$$$$
+\frac{\lambda^{3} \cdot C_{P}\left(\eta_{1}\right)}{\left(1+\varepsilon \cdot \cos \alpha_{T 1}\right)^{3}} \cdot \sin ^{2}\left(\pi-\vartheta_{1}\right) \text {. }
$$

Translate them from $r-\phi$ coordinate frame into $x-y$ coordinate frame, these are

$\left[\begin{array}{ll}K Y Y & K Y X \\ K X Y & K X X\end{array}\right]=\left[\begin{array}{cc}\cos \Phi & -\sin \Phi \\ \sin \Phi & \cos \Phi\end{array}\right] \cdot\left[\begin{array}{ll}K_{r r} & K_{r \varphi} \\ K_{\varphi r} & K_{\varphi \iota}\end{array}\right] \cdot\left[\begin{array}{cc}\cos \Phi & \sin \Phi \\ -\sin \Phi & \cos \Phi\end{array}\right]$

$\left[\begin{array}{ll}C Y Y & C Y X \\ C X Y & C X X\end{array}\right]=\left[\begin{array}{cc}\cos \Phi & -\sin \Phi \\ \sin \Phi & \cos \Phi\end{array}\right] \cdot\left[\begin{array}{cc}C_{r r} & C_{r \varphi} \\ C_{\varphi r} & C_{\varphi \iota}\end{array}\right] \cdot\left[\begin{array}{cc}\cos \Phi & \sin \Phi \\ -\sin \Phi & \cos \Phi\end{array}\right]$

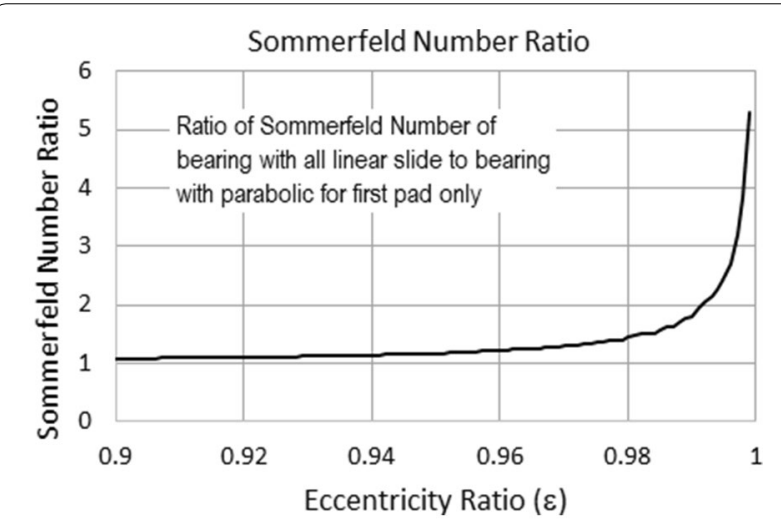

Figure 10 Ratio of Sommerfeld Number
The coefficients of stiffness $K Y Y, K Y X, K X Y$ and $K X X$ are non-dimensional. From equation group Eqs. (43) and (44), it can be seen that they only depend on location angles and the number of grooves. This means they are changing with different groove configurations. The same is applied to the damping coefficients. For purpose to make comparison with other available methods, here a new group of non-dimensional coefficients of stiffness and damping is defined as follows:

$$
\left[\begin{array}{ll}
K_{y y} & K_{y x} \\
K_{x y} & K_{x x}
\end{array}\right]=\left(\frac{2 B}{d}\right)^{2} \cdot S \cdot \pi \cdot\left[\begin{array}{ll}
K Y Y & K Y X \\
K X Y & K X X
\end{array}\right]
$$




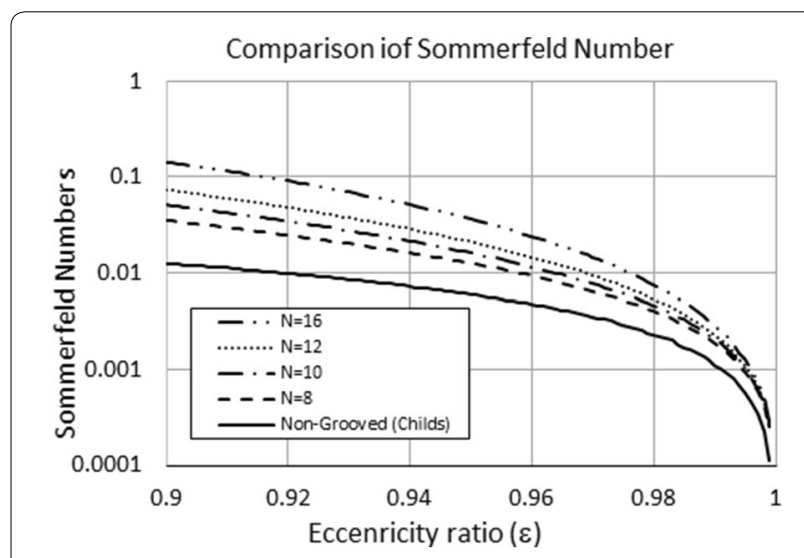

Figure 11 Sommerfeld Number of grooved bearings

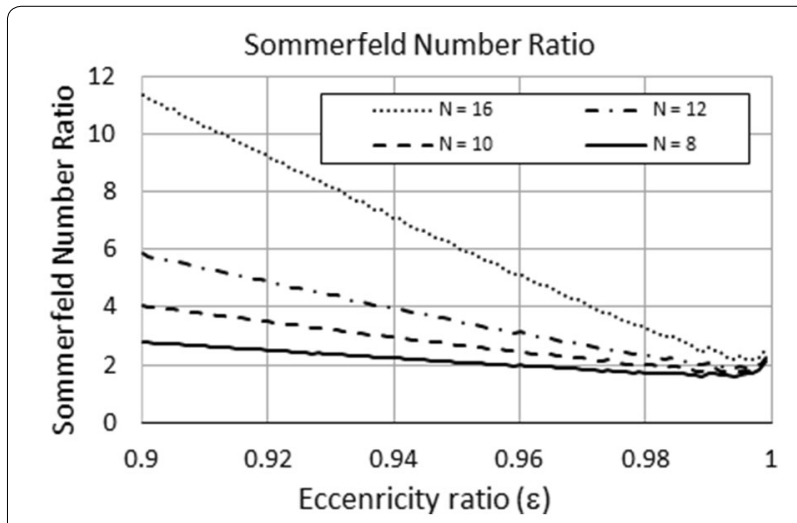

Figure 12 Ratio of Sommerfeld Number of grooved to non-grooved bearing

$$
\left[\begin{array}{ll}
C_{y y} & C_{y x} \\
C_{x y} & C_{x x}
\end{array}\right]=\left(\frac{2 B}{d}\right)^{3} \cdot S \cdot \pi \cdot\left[\begin{array}{ll}
C Y Y & C Y X \\
C X Y & C X X
\end{array}\right] .
$$

The Sommerfeld number in Eqs. (47) and (48) is the one defined by Eq. (42). The final dimensional coefficients of stiffness and damping are as follows:

$$
\begin{aligned}
& {\left[\begin{array}{ll}
k_{y y} & k_{y x} \\
k_{x y} & k_{x x}
\end{array}\right]=\frac{W_{o}}{c} \cdot\left[\begin{array}{ll}
K_{y y} & K_{y x} \\
K_{x y} & K_{x x}
\end{array}\right]=\frac{\mu \cdot V \cdot B^{2} \cdot L}{c^{3}}\left[\begin{array}{rr}
K Y Y & K Y X \\
K X Y & K X X
\end{array}\right],} \\
& (49) \\
& {\left[\begin{array}{ll}
c_{y y} & c_{y x} \\
c_{x y} & c_{x x}
\end{array}\right]=\frac{W_{o}}{c \cdot \Omega} \cdot\left[\begin{array}{ll}
C_{y y} & C_{y x} \\
C_{x y} & C_{x x}
\end{array}\right]=\frac{\mu \cdot B^{3} \cdot L}{c^{3}}\left[\begin{array}{ll}
C Y Y & C Y X \\
C X Y & C X X
\end{array}\right] .}
\end{aligned}
$$

The non-dimensional stiffness group in Eq. (47) and non-dimensional damping group in Eq. (48) is directly comparable with existing circular bearing results such as long and short bearing theory and others. In this paper, the stiffness and damping coefficients from Childs and Moes $[4,5]$ are of particular interest. They are considered to be accurate for non-grooved plain bearings and used for verifying the correctness of Eqs. (47) and (48).

Same as for non-dimensional coefficients of stiffness, the coefficients of damping have also been compared at same groove number and $L / D$ ratio. In Figures 13 and 14, the stiffness and damping coefficients by Childs and Moes was based on $L / D=2.0$ and for grooved bearings was based on groove number $=8$.

It is understandable that stiffness coefficient $K_{y y}$ for grooved bearing is slightly greater than that for nongrooved bearing. This is because the pressure is more concentrated on the area around of loading center due to grooves. The same reason may explain why the coefficient of stiffness $K_{x x}$ is lower than that of non-grooved bearing. The cross coefficient of stiffness $K_{y x}$ shows a different behavior from non-grooved bearing. Another noticeable characteristic is that the turning point of the cross-stiffness coefficient $K_{x y}$ into negative is shifted to lower eccentricity ratio.

The damping coefficient $C_{y y}$ is almost identical for both non-grooved bearing and grooved bearing in this particular geometrical condition. The coefficient $C_{x x}$ of grooved bearing is lower than that of non-grooved bearing. The cross-damping coefficient $C_{x y}=C_{y x}$ has larger difference for low and high eccentricity ratio and small difference for intermediate eccentricity ratio.

\section{Influence of the Number of Grooves}

As stated in previous section, the coefficients of stiffness and damping are not only a function of eccentricity ratio, but also the number of grooves. Figure 15 is a comparison between two bearings with 8 grooves and 12 grooves, respectively. The effect on the coefficients of stiffness is different. An increased groove number has an insignificant effect on $K_{y y}$ while it reduces $K_{y x}$. For crosscoefficient of stiffness $K_{x y}$, the increase of groove number shifts the turning point to negative to lower eccentricity ratio.

Figure 16 presents a comparison between the coefficients of damping for two bearings with 8 grooves and 12 grooves respectively. Again, the groove number has less effect on $C_{y y}$ while affecting other coefficients significantly.

\section{Conclusions}

This paper provides a new method to calculate the load capacity, the coefficients of stiffness and damping for water lubricated guide bearings with multi-axial grooves. The focus is on the effect of grooves and groove number. The paper doesn't include the effect of surface deformation. The result is an approximation and can be applied to water lubricated bearings made from hard polymers combined with lower pressure or other materials, such as 
Lignum Vitae wood and ceramics. The paper uses a socalled mixed scheme which means using parabolic slider for the first pad only, rest of the pads uses linear slider. The stiffness and damping of the grooved bearing was investigated considering groove effect. The coefficients of stiffness and damping demonstrated different characteristics from those with no grooves. Since the coefficients of stiffness and damping are function of eccentricity ratio and number of grooves, the effect of number of grooves was studied in great depth. It showed that the number of grooves has less effect on the coefficient $K_{y y}$ and $C_{y y}$ while it has a larger effect on other coefficients of stiffness and damping. Further research in considering surface deformation with using similar modeling could be an interesting subject.

\footnotetext{
Abbreviations

$A_{L}(\eta), A_{E}(\eta)$ or $A_{P}(\eta)$ : Non-dimensional location of static load center; $A_{L d}(\eta), A_{E d}(\eta)$ or $A_{P d}(\eta)$ : Non-dimensional location of dynamic load center; $B$ : Width of the slide bearing and the pad between two grooves $(\mathrm{m})$; $c$ : Radial bearing clearance $(\mathrm{m})$; $d$ : Shaft Diameter $(\mathrm{m}) ; \boldsymbol{e}$ : Eccentricity of the bearing $(\mathrm{m}) ; \boldsymbol{h}_{L 0}$ : Lubrication film thickness at leading edge of the slide bearing under steady operation $(\mathrm{m}) ; \boldsymbol{h}_{T 0}$ : Lubrication film thickness at trailing edge of the slide bearing under steady operation $(\mathrm{m})$; $\Delta h_{T i}(t)$ : Amount of dynamic squeeze of fluid film at trailing edge of pad ${ }^{\prime \prime}{ }^{\prime \prime}(\mathrm{m}) ; \Delta h_{T i}(t)$ : Dynamic squeeze velocity of fluid film at trailing edge of pad "i" $(\mathrm{m} / \mathrm{s}) ; \mathrm{L}$ : Bearing length, this is the length of bearing pad $(\mathrm{m}) ; \boldsymbol{x}^{*}$ :
}

\section{Authors' Information}

Guojun (Gary) Ren, born in 1959, graduated with M.Sc. in mechanical engineering from Technical University of Liaoning of China in 1984 and acquired his PhD in mechanical engineering from University of Stuttgart in Germany in 1996. He is currently employed in Thordon Bearings Inc. Canada. His research interest is Tribology and Rotating Machinery.

\section{Funding}

Not applicable.

\section{Availability of data and materials}

A program created with Mathcad to verify the calculation result is available. Its release is subject to employer approval. Datasets used during current study are available at Thordon Bearings Inc.

\section{Competing Interest}

The paper was created during employment at Thordon Bearings Inc. The author declares that the objective of this work was fully devoted to a solution of the particular problem in engineering and a better understanding its scientific nature in general. There is no any commercial or associated interest that represent a conflict of interest in connection with the work to a third party.

\section{Appendix: Parabolic Gap Sliding Bearing}

The procedure proposed by this paper uses a scheme by mixing different types of sliding bearings to build the entire circular bearing. One of the most important components is the parabolic gap sliding bearing. This appendix provides a procedure for deriving the four main functions, namely the load capacity, the location of load center, stiffness and damping.

Without loss of generality, the same coordinate system as shown in Figure 3 is used for this procedure. The shape of parabolic gap is expressed as

$$
h(x, t)=h_{T}(t) \cdot\left[1+(\eta-1) \cdot\left(\frac{x}{B}\right)^{2}\right], \quad-B \leq x \leq 0, \eta \geq 1 .
$$

Non-dimensional coordinate of sliding bearings, $x^{*}=x / B$; $N$ : number of bearing grooves, always designed with even number without loss of generality; $N_{s}$ : Shaft rotating speed in (r/s); $V$ : Surface velocity of shaft $(\mathrm{m} / \mathrm{s}) ; W_{o}$ : Loading force of the slide bearing under steady operation condition $(\mathrm{N}) ; W_{o, i}$ : $W_{o}$ (Referring to pad "i"); $W_{1}$ : Dynamic part of bearing load on top of $W_{o}(\mathrm{~N})$; $\alpha_{L i}$ : Location angle of leading edge of pad " $i$ "; $\alpha_{T i}$ : Location angle of trailing edge of pad " $i$ "; $\varepsilon$ : Eccentricity ratio of the entire bearing $(\varepsilon=e / c)$; $\Phi$ : Attitude angle of the bearing (rad); $\eta=h_{L 0} / h_{T 0}$ : Ratio of film thickness at leading edge to trailing edge); $\eta_{i}: \eta$ (Referring to pad " $i$ "); $\lambda$ : Part of first pad surface taking load (0 to 1.0); $\mu$ : Viscosity of lubricant, for water it is a constant (P.s); $\Omega$ : Angular velocity of shaft (1/s); $K_{y y}, K_{y x}, K_{x y}, K_{x x}$ : Non-dimensional coefficients of stiffness; $k_{y y}, k_{y x}, k_{x y}, k_{x x}$ : Dimensional coefficients of stiffness; $C_{y y}, C_{y x}, C_{x y}, C_{x x}$ : Non-dimensional coefficients of damping; $c_{y y}, c_{y x}, c_{x y}, c_{x x}$ : Dimensional coefficients of damping.

\section{Acknowledgements}

The employer provided a great support to this work and permits publishing this paper for a good will to general public and lubrication community.

\section{Authors' Contributions}

GGR was in charge of the whole trial and wrote the manuscript. All authors read and approved the final manuscript.
Introducing non-dimensional variables and parameters defined as follows:

$$
\begin{aligned}
& x^{*}=\frac{x}{B}, \quad \tau=\frac{V \cdot t}{B}, \quad h_{T}^{*}=\frac{h_{T}(t)}{h_{T 0}} \\
& h^{*}=h_{T}^{*} \cdot\left[1-(\eta-1) \cdot x^{*}\right], \quad p^{*}=\frac{\left(p-p_{g}\right) \cdot h_{T 0}^{2}}{\mu \cdot V \cdot B},
\end{aligned}
$$

where $p$ is the pressure over the pad with unit length, $p_{g}$ is the pressure in water grooves, $t$ is time.

The Reynolds Equation taking into consideration on dynamic squeezing film action is as follows:

$$
\frac{\partial}{\partial x}\left(h^{3} \cdot \frac{\partial p}{\partial x}\right)=6 \cdot \mu \cdot V \cdot \frac{\partial h}{\partial x}+12 \mu \cdot \frac{\partial h}{\partial t} .
$$

Insert non-dimensional variables Eq. (A2) into Eq. (A3), the Reynolds equation in non-dimensional form is 


$$
\frac{\partial}{\partial x^{*}}\left(h^{* 3} \cdot \frac{\partial p^{*}}{\partial x^{*}}\right)=6 \cdot \frac{\partial h^{*}}{\partial x^{*}}+12 \cdot \frac{\partial h^{*}}{\partial \tau} .
$$

Small perturbation method means to find a solution of Eq. (A4) not far from the steady state solution with a linearization approach. This implies to find a solution, such as

$$
\begin{aligned}
& p^{*}=p_{o}^{*}+p_{1}^{*} \cdot \delta \cdot e^{i \cdot \tau}, \\
& h_{T}^{*}=1+\delta \cdot e^{i \cdot \tau},
\end{aligned}
$$

where $p_{0}^{*}$ is non-dimensional pressure under steady operation, $p_{1}^{*}$ is perturbation amplitude of a dynamic pressure on top of the pressure under steady operation. In true sense $p_{1}^{*}$ is a coefficient of the non-dimensional dynamic pressure. $\delta$ is a small perturbation, which is a small number much less than 1.0. Its physical meaning is the ratio of amplitude change of film thickness to the minimum film thickness under steady operation. Insert Eqs. (A5) and (A6) into Eq. (A4), and equating the coefficients of zero order of " $\delta$ " on left and right side of Eq. (A4), it is resulted in an equation for pressure $p_{o}^{*}$

$$
\frac{\partial}{\partial x^{*}}\left(\left[1-(\eta-1) \cdot x^{*^{2}}\right]^{3} \cdot \frac{\partial p_{o}^{*}}{\partial x^{*}}\right)=12 \cdot(\eta-1) \cdot x^{*} .
$$

By the same token, by equating the coefficients of first order of " $\delta$ " on left and right side of Eq. (A4), the coefficient of dynamic pressure $p_{1}^{*}$ will fulfill following equation

$$
\frac{\partial}{\partial x^{*}}\left(\left[1-(\eta-1) \cdot x^{*^{2}}\right]^{3} \cdot \frac{\partial p_{1}^{*}}{\partial x^{*}}\right)=-24 \cdot(\eta-1) \cdot x^{*}+12 \cdot\left[1-(\eta-1) \cdot x^{*^{2}}\right] \cdot i, i=\sqrt{-1} .
$$

In this procedure, all other terms with orders equal to and higher than $\delta^{2}$ are neglected.

The boundary conditions for the non-dimensional pressure $p^{*}$ are

$$
p^{*}=0 \text { for } x^{*}=0 \text { and } x^{*}=-1 .
$$

To fulfill these conditions, the non-dimensional pressure on steady operation $p_{0}^{*}$ as well as the real and imaginary part of non-dimensional dynamic pressure all need to be zero on the boundaries. This is expressed as

$$
p_{0}^{*}=0 ; \quad p_{1}^{*}=p_{1, r}^{*}=p_{1, i}^{*}=0 \text { for } x=0 \text { and } x=-1 .
$$

First is to find the solution of Eq. (A7). By integrating twice of Eq. (A7), the non-dimensional pressure on steady operation is expressed in following form

$$
p_{o}^{*}=\frac{3}{4}\left\{\frac{\tan ^{-1} x^{*} \sqrt{\eta-1}}{\sqrt{\eta-1}}+\frac{x^{*^{3}}(\eta-1)-x^{*}}{\left[1+(\eta-1) \cdot x^{*^{2}}\right]^{2}}\right\}+\frac{C_{1}}{8}\left\{\frac{3 \tan ^{-1} x^{*} \sqrt{\eta-1}}{\sqrt{\eta-1}}+\frac{3 x^{*^{3}}(\eta-1)+5 x^{*}}{\left[1+(\eta-1) \cdot x^{*^{2}}\right]^{2}}\right\}+C_{2} \text {. }
$$

The boundary condition for $p_{0}^{*}$ requires $C_{2}=0$, and

$$
C_{1}=-2 \frac{\eta^{2} \tan ^{-1} \sqrt{\eta-1}+(\eta-2) \sqrt{\eta-1}}{\eta^{2} \tan ^{-1} \sqrt{\eta-1}+\left(\eta+\frac{2}{3}\right) \sqrt{\eta-1}}
$$

Insert $C_{1}$ into Eq. (A11), the final non-dimensional pressure on steady operation takes form as below
The load capacity function is the integration of the non-dimensional pressure (Eq. (A13))

$$
\Pi_{P}(\eta)=\frac{W_{o} \cdot h_{T o}^{2}}{\mu \cdot V \cdot B^{2} \cdot L}=\int_{-1}^{0} p_{o}^{*}\left(x^{*}, \eta\right) \cdot \mathrm{d} x^{*}
$$

$$
p_{o}^{*}=\frac{2}{\eta^{2} \tan ^{-1} \sqrt{\eta-1}+(\eta+2 / 3) \sqrt{\eta-1}} \times\left\{\tan ^{-1} x^{*} \sqrt{\eta-1}+\frac{x^{*}\left(x^{*^{2}}-1\right)(\eta-1)^{\frac{3}{2}}-x^{*} \eta^{2} \tan ^{-1} \sqrt{\eta-1}}{\left[1+(\eta-1) x^{*}\right]^{2}}\right\}(\mathrm{A}
$$



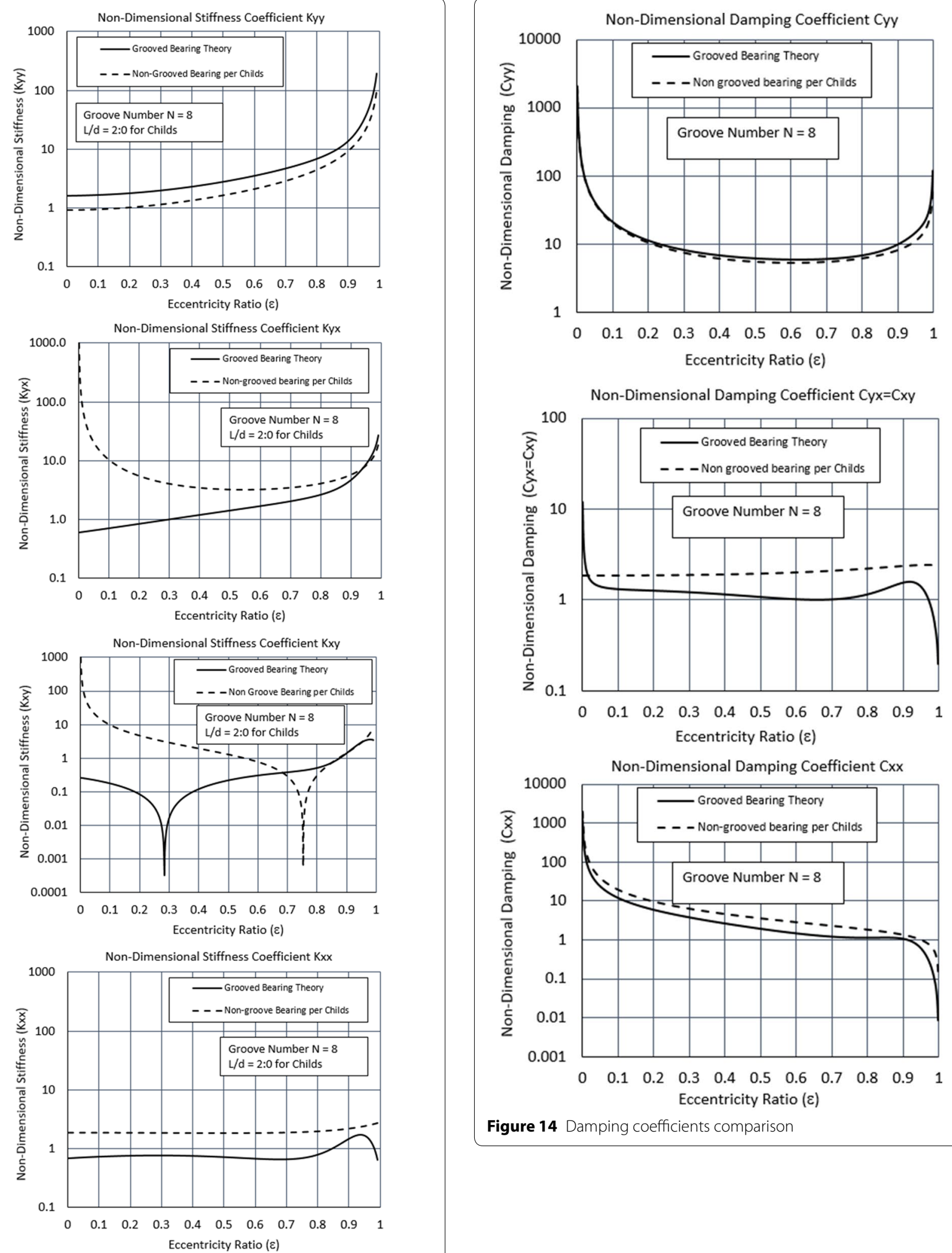

Figure 14 Damping coefficients comparison

Figure 13 Stiffness coefficients comparison 

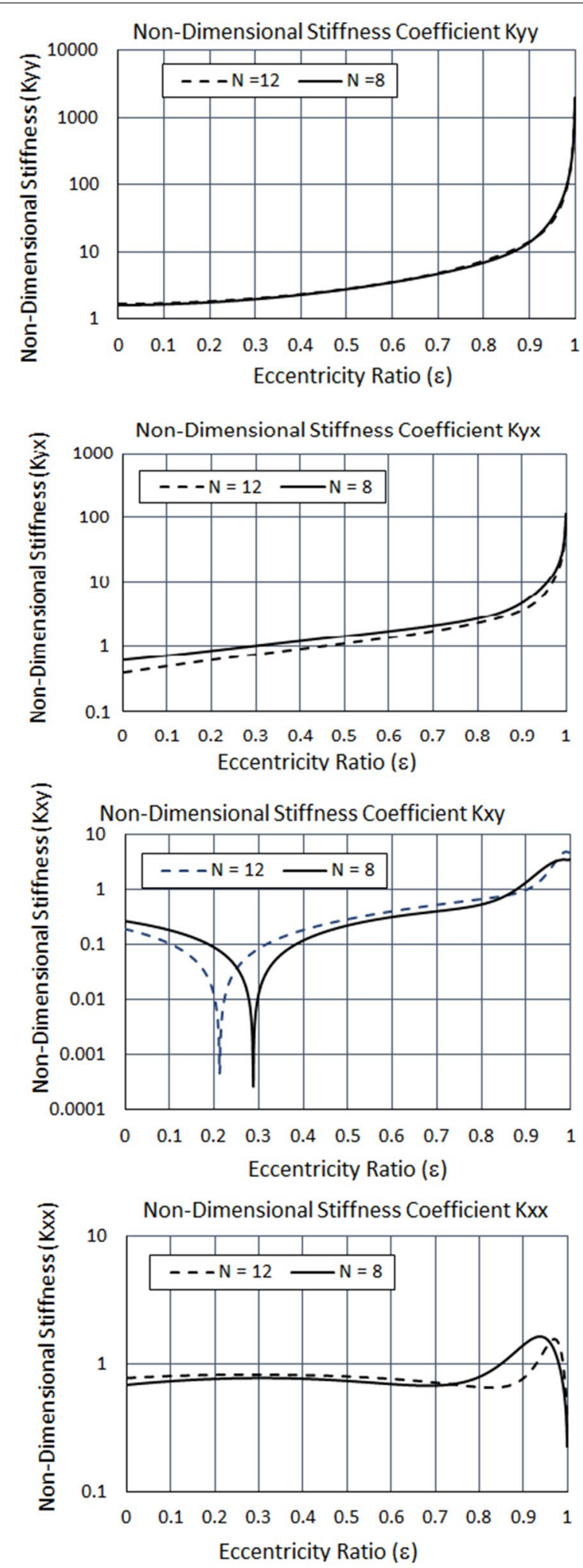

Figure 15 Influence of number of grooves on stiffness
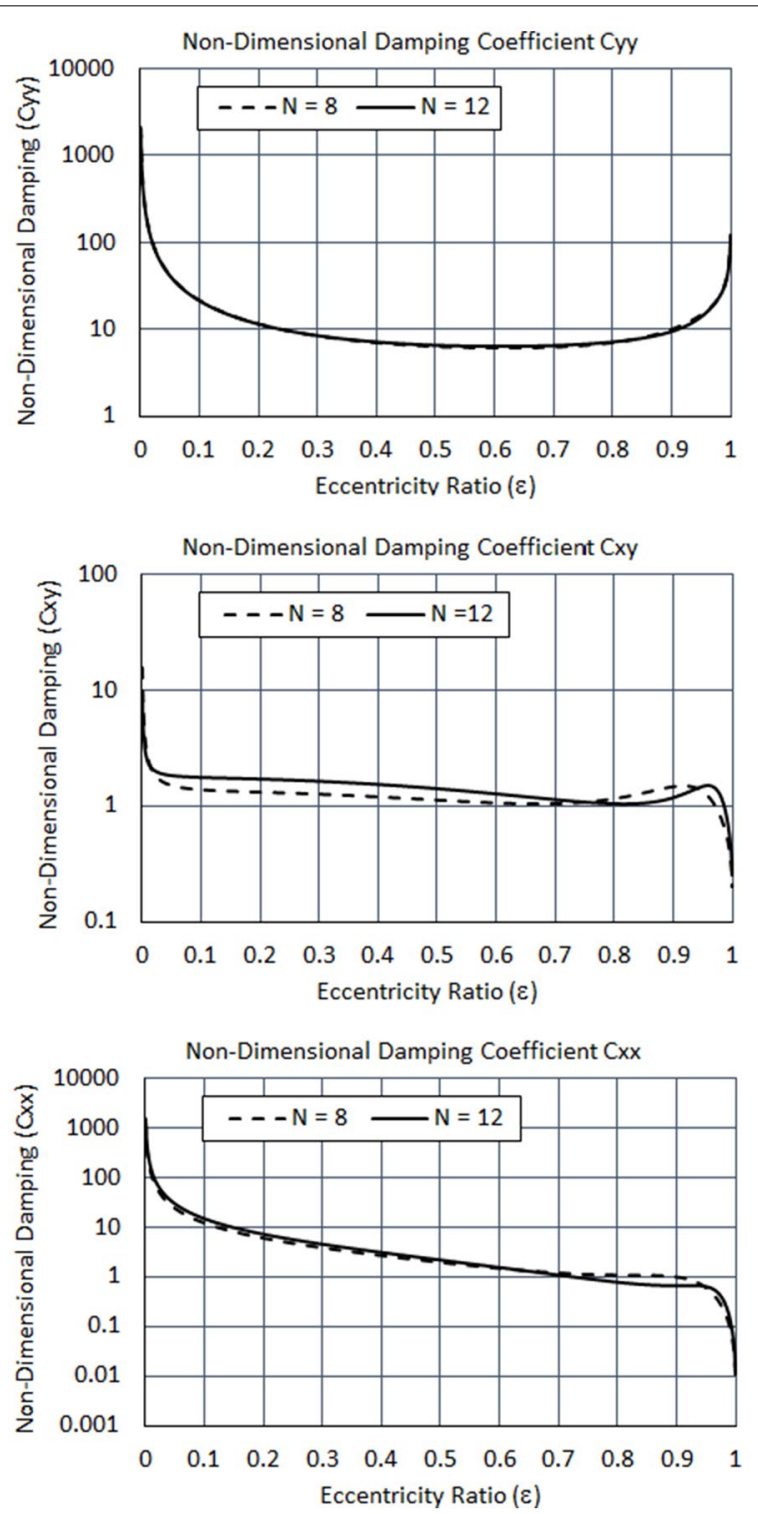

Figure 16 Influence of number of grooves on damping

$$
\Pi_{P}(\eta)=\frac{(\eta-2) \cdot \tan ^{-1} \sqrt{\eta-1}+\sqrt{\eta-1}}{\eta^{2} \tan ^{-1} \sqrt{\eta-1}+\left(\eta+\frac{2}{3}\right) \sqrt{\eta-1}} .
$$

It is interesting to notice that there is a similarity of right side of Eq. (A7) and the first term on right side of Eq. (A8). Since the solution of Eq. (A7) creates the load capacity function Eq. (A15), the first real term on the right side of Eq. (A8) must generate the stiffness function. This concludes that the stiffness function is just equal to two times of the load capacity function by amount, therefore

$$
K_{P}(\eta)=2 \frac{(\eta-2) \cdot \tan ^{-1} \sqrt{\eta-1}+\sqrt{\eta-1}}{\eta^{2} \tan ^{-1} \sqrt{\eta-1}+\left(\eta+\frac{2}{3}\right) \sqrt{\eta-1}} .
$$


Corresponding real part of non-dimensional dynamic pressure coefficient will be

$$
p_{1, r}^{*}=\frac{-4}{\eta^{2} \tan ^{-1} \sqrt{\eta-1}+(\eta+2 / 3) \sqrt{\eta-1}} \times\left\{\tan ^{-1} x^{*} \sqrt{\eta-1}+\frac{x^{*}\left(x^{*^{2}}-1\right)(\eta-1)^{\frac{3}{2}}-x^{*} \eta^{2} \tan ^{-1} \sqrt{\eta-1}}{\left[1+(\eta-1) x^{*^{2}}\right]^{2}}\right\}
$$

The next task is to find imaginary part of the nondimensional dynamic pressure coefficient $p_{1}^{*}$ which needs to fulfill following equation:

$$
\frac{\partial}{\partial x^{*}}\left(\left[1-(\eta-1) \cdot x^{*^{2}}\right]^{3} \cdot \frac{\partial p_{1, i}^{*}}{\partial x^{*}}\right)=12 \cdot\left[1-(\eta-1) \cdot x^{*^{2}}\right] .
$$

Following similar procedure to solve Eq. (A7), after integration twice of Eq. (A18), the imaginary part of nondimensional dynamic pressure coefficient is expressed with

And the location of load center for dynamic load only is calculated with

$$
A_{P d}(\eta)=1+\frac{\int_{-1}^{0} x \cdot \sqrt{p_{1 r}^{*^{2}}+p_{1 i}^{*^{2}}} \mathrm{~d} x}{\int_{-1}^{0} \sqrt{p_{1 r}^{*^{2}}+p_{1 i}^{*^{2}}} \mathrm{~d} x} .
$$

Since $p_{1 r}^{*}$ is two times of static pressure $p_{o}^{*}$ and has dominate amount in comparison to $p_{1 i}^{*}$, the value of Eq. (A24) is not very much different from the value from Eq.

$$
p_{1, i}^{*}=-2 \cdot \frac{x^{*^{2}}(\eta-1)+2}{\left[1+(\eta-1) \cdot x^{*^{2}}\right]^{2}(\eta-1)}+\frac{C_{3}}{8}\left\{\frac{3 \tan ^{-1} x^{*} \sqrt{\eta-1}}{\sqrt{\eta-1}}+\frac{3 x^{*^{3}}(\eta-1)+5 x^{*}}{\left[1+(\eta-1) \cdot x^{*^{2}}\right]^{2}}\right\}+C_{4} .
$$

Utilizing boundary condition to above equation, the two constants are
(A23). A ratio $R_{P}(\eta)=A_{P d}(\eta) / A_{P}(\eta)$ was defined for comparing the difference between Eqs. (A23) and (A24).

$$
C_{3}=\frac{8\left[4 \eta^{2}-2(\eta+1)\right]}{3 \eta^{2} \sqrt{\eta-1} \cdot \tan ^{-1} \sqrt{\eta-1}+(\eta-1)(3 \eta+2)}, C_{4}=\frac{4}{\eta-1} .
$$

Inserting them into Eq. (A19) and integrating it over from $x^{*}=-1$ to $x^{*}=0$, the damping function is as following:
Similarly this ratio is also defined for exponential and linear slider (see Figure 4d). This paper used static load center for Sommerfeld Number evaluation and dynamic

$$
\left.C_{P}(\eta)=-\int_{-1}^{0} p_{1, i}^{*}\left(x^{*}, \eta\right) \cdot \mathrm{d} x^{*}=\frac{2(2 \eta+1)}{3 \eta+2+\frac{3 \eta^{2}}{\sqrt{\eta-1}} \tan ^{-1} \sqrt{\eta-1}} \cdot\left(\frac{1}{\eta}+\frac{3 \tan ^{-1} \sqrt{\eta-1}}{\sqrt{\eta-1}}\right)-\frac{4 \eta-1}{\eta(\eta-1)}+\frac{3 \tan ^{-1} \sqrt{\eta}(\overline{\mathrm{A}} 21}{(\eta-1)^{\frac{1}{2}}}\right)
$$

The total pad pressure appears as complex function which is

$$
p^{*}=p_{o}^{*}+\left(p_{1 r}^{*}+i \cdot p_{1 i}^{*}\right) \cdot \delta \cdot e^{i \tau} .
$$

The location of load center under steady operation and dynamic vibration is slightly different. The location of load center for steady operation is calculated with

$$
A_{P}(\eta)=1+\frac{\int_{-1}^{0} x \cdot p_{o}^{*} \mathrm{~d} x}{\int_{-1}^{0} p_{o}^{*} \mathrm{~d} x} .
$$

load center for stiffness and damping evaluation for all three types of sliding bearings. The notion $A_{E d}$ and $A_{L d}$ presents the dynamic load center of exponential and linear slider respectively.

Received: 26 July 2019 Revised: 21 August 2020 Accepted: 12 October 2020

Published online: 28 October 2020

\section{References}

[1] G Ren, G Auger. Water film stiffness and damping analysis of water lubricated bearings with multiple axial grooves for hydro turbines. International Conference Hydro, 2016. Montreux, Switzerland, 10-12 Oct. 2016.

[2] Andreas Z. Szeri. Fluid film lubrication, theory and design. 1st ed. Cambridge: Cambridge University Press, 1998. 
[3] George B DuBois, Fred W Ocvirk. Analytical derivation and experimental evaluation of short-bearing approximation for full journal bearings. NACA Report 1157.

[4] Mircea Rades. Dynamics of Machinery II. Editura Printech, 2009: 99-102.

[5] D Childs, H Moes, HVan Leeuwen. Journal bearing impedance descriptions for rotor dynamic applications. Transactions of ASME, 1977:198.

[6] G Capone, V Agostino, D Guida. A finite length plain journal bearing theory. Transaction of ASME, 1994, 116: 648-653.

[7] R S Pai, R Pai. Stability of four-axial and six-axial grooved water-lubricated journal bearings under dynamic load. Proc. MechE Part J: J. Engineering Tribology, 2008, 222: 683-691.

[8] G Ren. Calculation of load capacity and water film thickness for fully grooved water lubricated main guide bearings for hydro turbines. Hydro Vision Russia, Moscow, March 3-5, 2015.

[9] Lahmar Mustapha, Ellagoune Salah, Sou-Said Benyebka. Elastohydrodynamic lubrication analysis of a compliant journal bearing considering static and dynamic deformations of the bearing liner. Tribology Transactions, 2010, 53: 349-368.

[10] C Liu, B Yao, G Cao, et al. Numerical calculation of composite water lubricated bearing considering effect of elastic deformation. IOP Conf. Series: Materials Science and Engineering, 2020, 772: 012114.

[11] Edward H Smith. On the design and lubrication of water-lubricated rubber, cutlass bearings operating in the soft EHL regime. Lubricants, 2020, 8: 75.

[12] G Zhou, J Wang, Y Han, et al. Study on the stiffness and damping coefficients of water lubricated rubber bearings with multiple grooves. Proceedings of the Institution of Mechanical Engineers, Part J: Journal of Engineering Tribology, 2016, 230(3): 323-335.

[13] Q Li, S Zhang, L Ma, et al. Stiffness and damping coefficients for journal bearing using the 3D transient flow calculation. Journal of Mechanical Science and Technology, 2017, 31(5): 2082-2091.

[14] X Liang, X Yan, Z Liu, et al. Effect of perturbation amplitudes on water film stiffness coefficients of water-lubricated plain journal bearings based on CFD-FSI methods. Proc. IMechE Part J. Journal of Tribology, 2018: 1-13.

[15] MV S Babu, A Rama Krishna, K N S Suman. Review of journal bearing material and current trends. American Journal of Material Science and Technology, 2015, 4(2): 72-83.

[16] Y Chen, Y Sun, Q He, et al. Elastohydodynamic behavior analysis of journal bearing using fluid-structure interaction considering cavitation. Arabian Journal for Science and Engineering, 2019, 44: 1305-1320.

[17] Elsayed K Elsayed, Alaa M A EL-Butch. A study on hydrodynamic water lubricated journal bearing. Engineering Research Journal, 2017, 153: M1-M15.

[18] Saeid Dousti, Paul Allaire, Timothy Dimond, et al. An extended Reynold equation applicable to high reduced Reynolds number of journal bearings. Tribology International, 2016, 102: 182-197.
[19] R Mallya, B S Shenoy, R S Pai, et al. Stability of water lubricated bearing using linear perturbation method under turbulent conditions. Pertanika J. Science and Technology, 2017, 25(3): 995-1008.

[20] KWu, G Zhou, X Mi, et al. Tribological and vibration properties of three different polymer material for water-lubricated bearings. Materials, 2020, 13: 3154.

[21] J Yang, Z Liu, X Liang, et al. Research on friction vibration of marine water lubricated rubber bearing. Tribology Online, Japanese Society of Tribologists, 2018, 13(3): 108-118.

[22] Wojciech Litwin. Properties comparison of rubber and three layer PTFE-NBR-Bronze water lubricated bearings with lubricating grooves along entire bush circumference based on experimental tests. Tribology International, 2015, 90: 404-411.

[23] XYe, J Wang, D Zhang, et al. Experimental research of journal orbit for water-lubricated bearing. Mathematical Problems in Engineering, 2016, 2016: 8361596

[24] T L Daugherty. Frictional characteristics of water-lubricated compliant surface stave bearings. ASLE, Transactions, 2008, 24(3): 293-301.

[25] C Chen, S Li, Z Lu, et al. Experimental study on material properties of bearing bush of water lubricated bearing. IOP Conf. Series: Materials Science and Engineering, 2020, 740: 012067.

[26] G C Brito Jr, R D Machado, A C Neto. Experimental estimation of journal bearing stiffness for damage detection in large hydrogenerators. Shock and Vibration, 2017: Article ID 4647868.

[27] N Wang, Q Meng, P Wang, et al. Experimental research on film pressure distribution of water-lubricated rubber bearing with multi-axial grooves. Journal of Fluids Engineering, Transactions of the ASME, 2013, 135.

[28] Wojciech Litwin. Experimental research on water lubricated three layer sliding bearing with lubrication grooves in the upper part of the bush and its comparison with a rubber bearing. Tribology International, 2015, 82: 153-161.

[29] Wojciech Litwin, Czeslaw Dymarski. Experimental research on waterlubricated marine stern tube bearings in conditions of improper lubrication and cooling causing rapid bush wear. Tribology International, 2016, 95: 449-455.

[30] T A Snyder, M J Braun. On the static and dynamic performance of compliant, water-lubricated sliding bearings; perturbed Reynolds equation vs. CFD-FSI based analysis methods. $18^{\text {th }}$ EDF/Pprime Workshop, EDF Lab Paris - Saclay, October 10-11, 2019.

[31] P Varpasuo, J Ahtiainen. Modeling of water lubricated bearing in hydro unit dynamic stability. HYDRO 2019, International Conference and Exhibition, Porto, Portugal, Allandega Porto Congress Center, 14-16 October, 2019 